\title{
Rete Ovarii Cystadenofibroma
}

National Cancer Institute

\section{Source}

National Cancer Institute. Rete Ovarii Cystadenofibroma. NCI Thesaurus. Code C40020.

An exceptionally rare cystadenofibroma that arises from the rete ovarii. 